\title{
On right-angled reflection groups in hyperbolic spaces
}

\author{
Leonid Potyagailo and Ernest Vinberg*
}

\begin{abstract}
We show that the right-angled hyperbolic polyhedra of finite volume in the hyperbolic space $\mathbb{H}^{n}$ may only exist if $n \leq 14$. We also provide a family of such polyhedra of dimensions $n=3,4, \ldots, 8$. We prove that for $n=3,4$ the members of this family have the minimal total number of hyperfaces and cusps among all hyperbolic right-angled polyhedra of the corresponding dimension. This fact is used in the proof of the main result.
\end{abstract}

Mathematics Subject Classification (2000). 20F55, 51F15, 57M07, 20F65, 57M50.

Keywords. Right-angled Coxeter polyhedra, reflection groups, hyperbolic orbifolds.

\section{Introduction}

An abstract Coxeter group $W$ is given by the following finite presentation:

$$
W=\left\langle S \mid\left(s_{i} s_{j}\right)^{m_{i j}}=1\right\rangle,
$$

where $m_{i j}=1$ if $i=j$ and $m_{i j} \in\{2,3, \ldots, \infty\}$ if $i \neq j$. By convention $m_{i j}=\infty$ means that there is no relation between $s_{i}$ and $s_{j}$. A Coxeter group $W$ is called right-angled if $m_{i j} \in\{1,2, \infty\}$.

Let $P$ be a convex polyhedron in the hyperbolic space $\mathbb{H}^{n}$ with dihedral angles of the form $\frac{\pi}{m}(m \in \mathbb{N})$ at all its (ordinary) $(n-2)$-dimensional faces. Then the group generated by the reflections in the $(n-1)$-dimensional faces (hyperfaces) of $P$ is a Coxeter group. Such a polyhedron $P$ is called a Coxeter polyhedron.

A polyhedron is called right-angled if all its dihedral angles are $\frac{\pi}{2}$. In this case the corresponding reflection group is a right-angled Coxeter group. Note that any face of a right-angled polyhedron is right-angled whereas a face of an arbitrary Coxeter polyhedron is not necessarily a Coxeter polyhedron. The following is the main result of the present paper:

Theorem. Right-angled Coxeter polyhedra of finite volume may exist in $\mathbb{H}^{n}$ only if $n \leq 14$.

\footnotetext{
${ }^{*}$ The work of the second author was partly supported by the RFBR grant 01-01-00756.
} 
Recall that E. Vinberg [Vi] proved that compact Coxeter polyhedra in $\mathbb{H}^{n}$ may exist only if $n \leq 29$. Examples are known only up to $n=8$. M. Prokhorov [Pr] proved that non-compact Coxeter polyhedra of finite volume may exist only if $n \leq 995$; examples are known only up to $n=21$ [Bor].

There are some strong restrictions on the combinatorial structure of Coxeter polyhedra arising from the property that all their dihedral angles do not exceed $\frac{\pi}{2}$. Polyhedra having the latter property are called acute-angled. It is known (see, e.g., [AVS88]) that any $k$-dimensional face of an acute-angled polyhedron $P \subset \mathbb{H}^{n}$ belongs only to $n-k$ hyperfaces. In particular, any (ordinary) vertex belongs only to $n$ hyperfaces, so the local combinatorial structure of $P$ at any vertex is the same as that of a simplicial cone. The local combinatorial structure of $P$ at any vertex at infinity is the same as that of a cone over a direct product of simplices; if $P$ is right-angled, these simplices must be one-dimensional (so their product is a $(n-1)$-dimensional parallelepiped).

An $n$-dimensional combinatorial polytope is called simple if any of its vertices belongs only to $n$ hyperfaces, and simple at edges if any of its edges belongs only to $n-1$ hyperfaces. According to the above, any compact acute-angled polyhedron in $\mathbb{H}^{n}$ is simple, and any acute-angled polyhedron of finite volume (with vertices at infinity added) is simple at edges.

It is known that compact right-angled polyhedra do not exist if $n>4$. This follows from the Nikulin inequality [N] for the average number $a_{k}^{l}$ of $l$-dimensional faces of a $k$-dimensional face of a simple polytope. It implies that in dimension $n>4$ any simple polyhedron $P$ must have a quadrilateral or triangular 2-dimensional face, which is impossible if $P$ is right-angled (see the next section). The estimate $n \leq 4$ is exact as there exist right-angled compact polyhedra in $\mathbb{H}^{4}$.

In the finite volume case some vertices can be at infinity so the above method does not work. To prove the Theorem we will obtain a lower bound for the number of 4-dimensional faces of a 5-dimensional right-angled polyhedron. Then the theorem will follow from Khovanskii's result [Kh] which generalizes the Nikulin inequality to polytopes that are simple at edges. Contrary to the compact case, our estimate $n \leq 14$ may be not exact as examples of right-angled polyhedra of finite volume are known only up to $n=8$ (we provide some of them in Section 3). Note also that our result cannot be much improved using the same method since the Nikulin-Khovanskii inequality is applied only for $l<k \leq\left[\frac{n}{2}\right]$ and, on the other hand, our estimates for the minimal number of hyperfaces of a low dimensional right-angled polyhedron are optimal.

Note that recently T. Januszkiewicz and J. Swiatkowki [JS] proved that there exist abstract word hyperbolic right-angled Coxeter groups of any virtual cohomological dimension.

Right-angled Coxeter groups in the hyperbolic spaces are known to have some strong group-theoretical properties. For a finitely generated abstract group $G$, let us call a subgroup $H \subset G$ closed if it is an intersection of subgroups of finite index or, 
equivalently, if it is closed in the topology defined by the subgroups of finite index. P. Scott [Sc] proved that if $G \subset \mathrm{Isom}_{+} \mathbb{H}^{n}$ is a discrete group commensurable with a co-compact right-angled reflection group, then any geometrically finite subgroup of $G$ is closed. (In fact he considered only the case $n=2$ but the idea of his proof works for any $n$.) I. Agol, D. Long and A. Reid [ALR] extended this theorem to groups commensurable with co-finite right-angled reflection groups.

Acknowledgments. This work was mostly done during the stay of the second author at the University of Lille 1 in June of 2002. He thanks this university for hospitality. The first author is grateful to A. Vesnin for helpful remarks. We thank the referee for pointing out some inaccuracies in the original version of the paper.

\section{Compact right-angled polyhedra}

For brevity, let us call $k$-dimensional faces of a polyhedron $P$ simply $k$-faces. Denote the number of $k$-faces by $a_{k}=a_{k}(P)$. Following V. Nikulin [N], consider the average number $a_{k}^{l}$ of $l$-faces of a $k$-face:

$$
a_{k}^{l}=\frac{1}{a_{k}} \sum_{\operatorname{dim} F=k} a_{l}(F)
$$

where $F$ runs over all $k$-faces of $P$. One of the main ingredients for proving the Theorem is the following Nikulin inequality $[\mathrm{N}]$ :

$$
a_{k}^{l}<C_{n-l}^{n-k} \frac{C_{\left[\frac{n}{2}\right]}^{l}+C_{\left[\frac{n+1}{2}\right]}^{l}}{C_{\left[\frac{n}{2}\right]}^{k}+C_{\left[\frac{n+1}{2}\right]}^{k}}
$$

for $l<k \leq\left[\frac{n}{2}\right]$.

For the sake of completeness we provide the proof of the following known

Proposition ([Vi]). There are no compact right-angled polyhedra in $\mathbb{H}^{n}$ for $n>4$.

Proof. By the Nikulin inequality we obtain

$$
a_{2}^{1}< \begin{cases}\frac{4(n-1)}{n-2} & \text { if } n \text { is even, } \\ \frac{4 n}{n-1} & \text { if } n \text { is odd. }\end{cases}
$$

For a compact right-angled polyhedron, every 2 -face being also right-angled has at least 5 sides. Thus $a_{2}^{1} \geq 5$ and the above inequality implies $n \leq 4$.

Remarks. a) The maximal dimension $n=4$ given by the proposition is attained: indeed, there exists a regular compact right-angled polyhedron in $\mathbb{H}^{4}$ with 120 dodecahedral hyperfaces [Cox], [D], [VS88].

b) There exist infinitely many compact right-angled polyhedra in $\mathbb{H}^{4}$ [VS88]. 
Question. Is it true that the least number of hyperfaces of a compact right-angled polyhedron in $\mathbb{H}^{4}$ is 120 ?

\section{A series of non-compact right-angled polyhedra}

Let us now describe one known series of right-angled hyperbolic polyhedra of finite volume [D]. We list below (Figure 1) the Coxeter diagrams $\Sigma^{n}$ of some non-compact Coxeter simplices $\Delta^{n}$ of finite volume in $\mathbb{H}^{n}$ for $n=3,4,5,6,7,8$. These are some of the so-called quasi-Lannér diagrams [VS88], pp. 206-207. The group generated by the reflections in hyperfaces of $\Delta^{n}$ will be denoted by $G^{n}$.

Let us introduce the following notation:

$F_{k}^{n}$ : the hyperface of $\Delta^{n}$ corresponding to the vertex $k$ of the diagram,

$O_{k}^{n}$ : the vertex (or cusp) of $\Delta^{n}$ opposite to $F_{k}^{n}$,

$\Sigma_{k}^{n}$ : the subdiagram of $\Sigma^{n}$ obtained by deleting the vertex $k$,

$G_{k}^{n}$ : the stabilizer of $O_{k}^{n}$, i.e. the group generated by the reflections in all the hyperfaces of $\Delta^{n}$ but $F_{k}^{n}$; its Coxeter diagram is $\Sigma_{k}^{n}$.
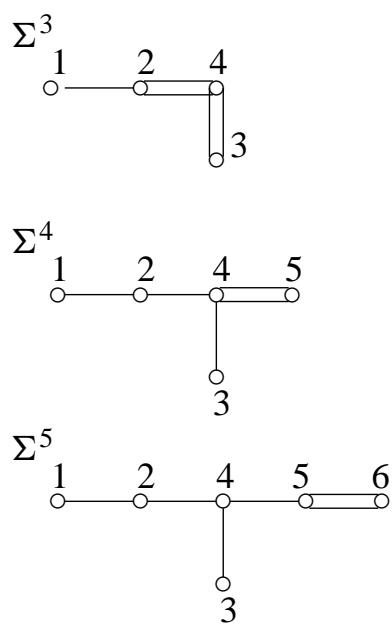
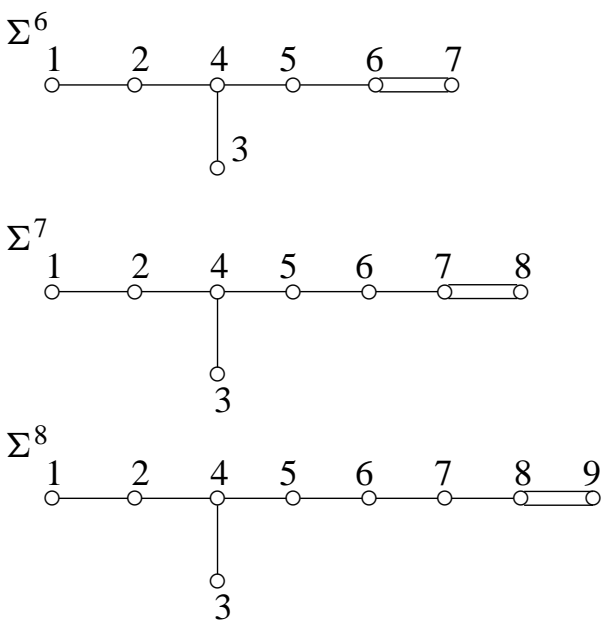

Figure 1

All the diagrams $\Sigma_{k}^{n}$ but $\Sigma_{1}^{n}$ are elliptic, while $\Sigma_{1}^{n}$ is parabolic. (See, e.g., [VS88] for the lists of elliptic and parabolic Coxeter diagrams.) Recall that elliptic (resp. parabolic) diagrams correspond to finite (resp. affine) Coxeter groups. Thus, $O_{1}^{n}$ is the only cusp of $\Delta^{n}$.

One can note that the hyperface $F_{n+1}^{n}$ of $\Delta^{n}$ forms only angles $\frac{\pi}{2}$ and $\frac{\pi}{4}$ with the other hyperfaces and the group $G_{n+1}^{n}$ is finite. Thus the translates of $\Delta^{n}$ under $G_{n+1}^{n}$ fit 
together at $O_{n+1}^{n}$ to give a right-angled polyhedron $P^{n}$ of finite volume. Its boundary is composed of the translates of $F_{n+1}^{n}$ (some of them lying in one hyperplane).

Let us describe the polyhedron $P^{3}$. Applying to $\Delta^{3}$ the group of order 6 generated by the reflections in $F_{1}^{3}$ and $F_{2}^{3}$, we get a tetrahedron with 3 cusps whose faces passing through the ordinary vertex are mutually perpendicular and form angles $\frac{\pi}{4}$ with the remaining face (which contains all the cusps). Applying to this tetrahedron the group of order 2 generated by the reflection in $F_{3}^{3}$, we get $P^{3}$ (see Figure 2, where the cusps are marked by small circles).

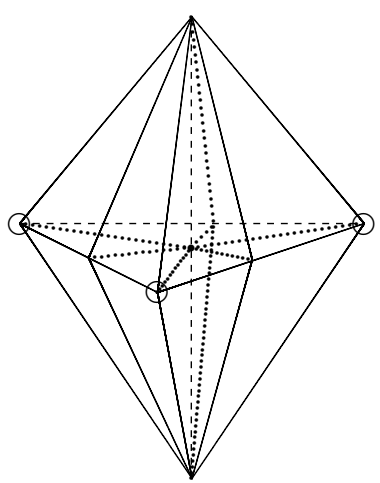

Figure 2

Any face of $P^{3}$ is composed of two copies of $F_{4}^{3}$ and is a triangle with two cusps and an angle $\frac{\pi}{2}$ at the ordinary vertex. Hence, $F_{4}^{3}$ is a triangle with one cusp and angles $\frac{\pi}{2}$ and $\frac{\pi}{4}$ at the ordinary vertices.

Proposition 3.1. For $n=4,5,6,7,8$, all the hyperfaces of the polyhedron $P^{n}$ are polyhedra $P^{n-1}$. The numbers of hyperfaces and cusps of the polyhedra $P^{n}$ are given in the following table:

\begin{tabular}{|c|c|c|}
\hline & Number of hyperfaces & Number of cusps \\
\hline$P^{3}$ & 6 & 3 \\
\hline$P^{4}$ & 10 & 5 \\
\hline$P^{5}$ & 16 & 10 \\
\hline$P^{6}$ & 27 & 27 \\
\hline$P^{7}$ & 56 & 126 \\
\hline$P^{8}$ & 240 & 2160 \\
\hline
\end{tabular}

Proof. In addition to the above notation, let us introduce the following one:

$$
F_{k l}^{n}=F_{k}^{n} \cap F_{l}^{n} \text {, }
$$


$\Sigma_{k l}^{n}$ : the subdiagram of $\Sigma^{n}$ obtained by deleting the vertices $k$ and $l$,

$G_{k l}^{n}=G_{k}^{n} \cap G_{l}^{n}$; this is a reflection group whose Coxeter diagram is $\Sigma_{k l}^{n}$. All the diagrams $\Sigma_{k l}^{n}$ are elliptic and the groups $G_{k l}^{n}$ are finite.

The hyperface $F$ of $P^{n}$ containing $F_{n+1}^{n}$ is composed of the translates of $F_{n+1}^{n}$ under the subgroup $H^{n}$ of $G_{n+1}^{n}$ generated by the reflections in hyperfaces $F_{k}^{n}$ perpendicular to $F_{n+1}^{n}$. All the hyperfaces of $P^{n}$ are the translates of $F$ under $G_{n+1}^{n}$, hence

$$
\#\left(\text { hyperfaces of } P^{n}\right)=\frac{\left|G_{n+1}^{n}\right|}{\left|H^{n}\right|} .
$$

For $n>3$, all the faces $F_{k}^{n}, k=1, \ldots, n-1$, are perpendicular to $F_{n+1}^{n}$, so $H^{n}=$ $G_{n, n+1}^{n}$, whence

$$
\text { \#(hyperfaces of } \left.P^{n}\right)=\frac{\left|G_{n+1}^{n}\right|}{\left|G_{n, n+1}^{n}\right|} .
$$

The orders of the finite Coxeter groups being known, this allows us to calculate the numbers of hyperfaces of the polyhedra $P^{n}$.

In a similar way, one can calculate the number of cusps of $P^{n}$. They constitute just the orbit of $O_{1}^{n}$ under $G_{n+1}^{n}$. The stabilizer is $G_{1, n+1}^{n}$, hence

$$
\#\left(\operatorname{cusps} \text { of } P^{n}\right)=\frac{\left|G_{n+1}^{n}\right|}{\left|G_{1, n+1}^{n}\right|} .
$$

Let us now prove that for $n>3$ the face $F_{n+1}^{n}$ of the simplex $\Delta^{n}$ is the simplex $\Delta^{n-1}$, which will imply that the face $F$ of the polyhedron $P^{n}$ is the polyhedron $P^{n-1}$.

Obviously, if $F_{k}^{n}$ and $F_{l}^{n}$ are perpendicular to $F_{n+1}^{n}$, then the angle between the corresponding faces $F_{k, n+1}^{n}$ and $F_{l, n+1}^{n}$ of the simplex $F_{n+1}^{n}$ is equal to the angle between $F_{k}^{n}$ and $F_{l}^{n}$.

If $F_{k}^{n}$ is perpendicular to $F_{n+1}^{n}$, while the angle between $F_{l}^{n}$ and $F_{n+1}^{n}$ is $\frac{\pi}{4}$, then, as one can observe in Figure 1, the angle between $F_{k}^{n}$ and $F_{l}^{n}$ is $\alpha=\frac{\pi}{2}$ or $\frac{\pi}{3}$. Let us find the angle $\beta$ between $F_{k, n+1}^{n}$ and $F_{l, n+1}^{n}$. Considering a 3-dimensional orthogonal section, we reduce the problem to the following one: given a tetrahedral angle with dihedral angles $\frac{\pi}{2}, \frac{\pi}{4}$ and $\alpha$, find its plane angle $\beta$ opposite to $\alpha$. Clearly, if $\alpha=\frac{\pi}{2}$. then $\beta=\frac{\pi}{2}$. If $\alpha=\frac{\pi}{3}$. then our tetrahedral angle is just the angle at the vertex $O_{3}^{3}$ of the tetrahedron $\Delta^{3}$ and, as we proved above, $\beta=\frac{\pi}{4}$.

Thus, $F_{n+1}^{n}$ is again a Coxeter simplex and its diagram is obtained from $\Sigma^{n}$ by deleting the vertex $n+1$ and replacing all simple edges joining the vertex $n$ with other vertices by double edges. (For $n=4$, there are two such edges; in all the other cases, there is only one.) One can observe that in such a way we get just the diagram $\Sigma^{n-1}$.

Now the hyperface $F$ of $P^{n}$ is obtained by fitting together the translates of $F_{n+1}^{n}$ under $H^{n}$. As $F_{n+1}^{n}$ is the simplex $\Delta^{n-1}$ and $H^{n}=G_{n}^{n-1}, F$ (and, hence, each hyperface of $P^{n}$ ) is the polyhedron $P^{n-1}$. 


\section{Proof of the theorem}

In this section, the word "polyhedron" always means "polyhedron of finite volume", i.e. a convex hull of finitely many ordinary points and points at infinity. The latter ones are called cusps of the polyhedron. We tacitly add them to the polyhedron and to its faces, so the expression "the faces $F_{1}, \ldots, F_{k}$ intersect" means that the faces $F_{1}, \ldots, F_{k}$ have a common ordinary point or their closures in the compactification of $\mathbb{H}^{n}$ have a common point at infinity. For a hyperface $F$ of a polyhedron, we denote by $H(F)$ the closure of the hyperplane containing $F$ in the compactification of $\mathbb{H}^{n}$.

Recall that two hyperplanes of $\mathbb{H}^{n}$ are called parallel if they do not intersect but their closures have a (single) point at infinity in common. We shall call two hyperfaces $F_{1}$ and $F_{2}$ of a polyhedron parallel if the hyperplanes containing them are parallel. It follows from the local combinatorial structure of right-angled polyhedra (see the introduction) that for any hyperface of such a polyhedron passing through a cusp $p$ there is a unique parallel hyperface passing through $p$.

The following properties will be used in the subsequent proof.

Proposition 4.1. Let $F_{1}, F_{2}, \ldots$ be hyperfaces of a right-angled polyhedron. Then

(a) if $H\left(F_{1}\right)$ and $H\left(F_{2}\right)$ intersect, then $F_{1}$ and $F_{2}$ intersect; in particular, if $F_{1}$ and $F_{2}$ are parallel, then they meet at a cusp;

(b) if $F_{1}, F_{2}, F_{3}$ are pairwise mutually adjacent, then they meet at an $(n-3)$-dimensional face;

(c) if $F_{1}$ and $F_{2}$ are parallel and $F_{3}$ is adjacent to them, then $F_{1}, F_{2}, F_{3}$ meet at a cusp;

(d) if $F_{1}$ and $F_{2}$ are parallel and $F_{3}$ and $F_{4}$ are adjacent to them, then $F_{1}, F_{2}, F_{3}, F_{4}$ meet at a cusp.

Proof. It is proved in [A70] (see also [AVS88]) that, for any hyperfaces $F_{1}, \ldots, F_{k}$ of an acute-angled polyhedron

$$
\operatorname{dim} F_{1} \cap \cdots \cap F_{k}=\operatorname{dim} H\left(F_{1}\right) \cap \cdots \cap H\left(F_{k}\right),
$$

where the dimension of a point at infinity is assumed to be -1 , while the dimension of the empty set is $-\infty$. This proves (a).

To show (c) note that the hyperfaces $H\left(F_{i}\right)(i=1,2,3)$ must meet in a cusp, for otherwise in a 2-dimensional orthogonal plane there would exist a triangle with two right angles and one zero angle which is impossible. Thus (c) follows now from the dimension identity.

To prove (b) note similarly that three mutually perpendicular hyperplanes must intersect in a $(n-3)$-dimensional plane. Indeed, if there were no common intersection between them, in the orthogonal 2-dimensional plane one would obtain a right-angled triangle which is not possible. The dimension identity implies now (b). 
To prove (d), note that by (c) both $F_{1}, F_{2}, F_{3}$ and $F_{1}, F_{2}, F_{4}$ meet at a cusp. But these two cusps must coincide, because $F_{1}$ and $F_{2}$ have only one cusp in common.

Let us now obtain lower bounds for some combinations of the numbers of hyperfaces and cusps of a right-angled polyhedron $P \subset \mathbb{H}^{n}$ for small $n$.

Recall that $a_{k}=a_{k}(P)$ denotes the number of $k$-faces of $P$. In particular, $a_{0}=$ $a_{0}(P)$ is the total number of ordinary vertices and cusps of $P$. The number of cusps will be denoted by $c=c(P)$.

Case $\boldsymbol{n}=2$. Since the sum of exterior angles of a hyperbolic polygon is greater than $2 \pi$, for a right-angled polygon $P$ we get

$$
a_{1}+c \geq 5 \text {. }
$$

The difference $a_{1}+c-5$ will be called the excess of $P$ and denoted by $e=\operatorname{ex}(P)$.

Case $n=3$. For each face $F$ of a right-angled polyhedron $P \subset \mathbb{H}^{3}$ we have

$$
a_{1}(F)+c(F)=5+\operatorname{ex}(F) .
$$

Summing over all $F$ and taking into account that each edge of $P$ belongs to 2 faces and each cusp belongs to 4 faces, we get

$$
2 a_{1}+4 c=5 a_{2}+\sum_{F} \operatorname{ex}(F) .
$$

On the other hand, eliminating $a_{0}$ from the Euler equation $a_{0}-a_{1}+a_{2}=2$ and the obvious equation $2 a_{1}=3 a_{0}+c$ gives

$$
a_{1}+c=3 a_{2}-6 .
$$

Substituting this into (2), we finally obtain

$$
a_{2}+2 c=12+\sum_{F} \operatorname{ex}(F) \geq 12 .
$$

At the same time

$$
a_{2} \geq 6 .
$$

Indeed, if $P$ has no cusps, this follows from (4); if $P$ has at least one cusp and $a_{2}<6$, then $P$ is a quadrilateral pyramid (whose apex is at infinity), which is obviously impossible.

It follows from (4) and (5) that

$$
a_{2}+c \geq 9 .
$$

Note that all the estimates (4)-(6) are attained for $P^{3}$ (see Figure 2). 
Case $n=4$. Let $P$ be a right-angled polyhedron in $\mathbb{H}^{4}$. Take any hyperface $F$ of it. There are $a_{2}(F)$ hyperfaces adjacent to $F$ and, for each cusp of $F$, there is an extra hyperface having only this cusp in common with $F$. Together with $F$, this gives at least $1+a_{2}(F)+c(F)$ hyperfaces. So (6) implies

$$
a_{3} \geq 10 \text {. }
$$

We need, however, a more subtle inequality:

$$
a_{3}+c \geq 15 .
$$

To prove it, take again any hyperface $F$ of $P$. There are at least $1+a_{2}(F)+c(F)$ hyperfaces meeting $F$ and at least $c(F)$ cusps, so $a_{3}+c \geq 1+a_{2}(F)+2 c(F)$. If $a_{2}(F)+2 c(F) \geq 14$, then (8) follows.

By (4) we have $a_{2}(F)+2 c(F) \geq 12$.

Let $a_{2}(F)+2 c(F)=13$. Then (4) implies that all but one 2 -faces of $F$ have zero excess. Let $f$ be a 2 -face of $F$ with zero excess, i.e. $a_{1}(f)+c(f)=5$. Since $c(f) \leq 2$, we have

$$
1+a_{1}(f)+2 c(f) \leq 8 .
$$

Let $F^{\prime}$ be the hyperface of $P$ adjacent to $F$ along $f$. By (6) we have that $a_{2}\left(F^{\prime}\right)+c\left(F^{\prime}\right) \geq 9$. Comparing this with (9), we see that $F^{\prime}$ must have either a 2 -face $f^{\prime}$ not intersecting $F$, or a cusp beyond $F$. In the first case the hyperface adjacent to $F^{\prime}$ along $f^{\prime}$ does not intersect $F$ by Proposition 4.1 (b), (c). So in both cases (8) holds.

Let $a_{2}(F)+2 c(F)=12$. Then (4) implies that all 2-faces of $F$ have zero excess. Let $f$ be any of them. Then $f$ is a triangle with two cusps, or a quadrilateral with one cusp, or else a pentagon without cusps. If $f$ is not a triangle, then

$$
1+a_{1}(f)+2 c(f) \leq 7 .
$$

As above, consider the hyperface $F^{\prime}$ of $P$ adjacent to $F$ along $f$. Then (10) implies that $F^{\prime}$ has at least two 2 -faces not intersecting $F$ or cusps beyond $F$, whence again (8) follows.

Let finally all 2-faces of $F$ be triangles with two cusps. Take any parallel 2-faces $f_{1}$ and $f_{2}$ of $F$, and let $F_{1}$ and $F_{2}$ be the hyperfaces of $P$ adjacent to $F$ along $f_{1}$ and $f_{2}$ respectively. By the above each of them must have either a 2 -face not intersecting $F$ or a cusp beyond $F$. If these are two 2-faces, then the hyperfaces of $P$ adjacent to $F_{1}$ and $F_{2}$ along them, cannot coincide by Proposition 4.1(d). If these are two cusps, then they cannot coincide as $F_{1}$ and $F_{2}$ are parallel at a cusp of $F$. So in all the cases (8) holds.

Note that both the estimates (7) and (8) are attained for the polyhedron $P^{4}$ constructed in Section 3. 
Case $\boldsymbol{n}=\mathbf{5}$. Let $P$ be a right-angled polyhedron in $\mathbb{H}^{5}$. Take any hyperface $F$ of it. There are $a_{3}(F)$ hyperfaces adjacent to $F$ and, for each cusp of $F$, there is an extra hyperface having only this cusp in common with $F$. Together with $F$, this gives at least $1+a_{3}(F)+c(F)$ hyperfaces. So (8) implies

$$
a_{4} \geq 16 \text {. }
$$

This estimate is attained for the polyhedron $P^{5}$ constructed in Section 3.

Proof of the theorem. Let $P \subset \mathbb{H}^{n}$ be a right-angled Coxeter polyhedron. The Nikulin-Khovanskii inequality [N], [Kh] gives for the average number $a_{5}^{4}$ of 4-faces of a 5-face of $P$ :

$$
a_{5}^{4}< \begin{cases}\frac{10(n-4)}{n-8} & \text { if } n \text { is even } \\ \frac{10(n-3)}{n-7} & \text { if } n \text { is odd }\end{cases}
$$

On the other hand, it follows from the above that $a_{5}^{4} \geq 16$. In both cases this means that $n \leq 14$.

We finish this section with some questions and remarks.

Questions. 1) Is it true that the least number of hyperfaces of a right-angled 6dimensional polyhedron is 27 (which is attained for $P^{6}$ )?

2) Do there exist right-angled polyhedra in $\mathbb{H}^{n}$ for $n=9,10,11,12,13,14$ ?

Remark. By a similar argument, a positive answer to the first question would exclude the dimensions 13 and 14 .

\section{References}

[ALR] I. Agol, D. D. Long and A. W. Reid, The Bianchi groups are separable on geometrically finite subgroups. Ann. of Math. (2) 153 (2001), no. 3, 599-621. Zbl 02081321 MR 1836283

[AVS88] D. V. Alekseevskij, E. B. Vinberg and A. S. Solodovnikov, Geometry of spaces of constant curvature. Itogi Nauki Tekh., Ser. Sovrem. Probl. Mat., Fundam. Napravleniya 29 (1988), 5-146; English transl. in Geometry II. Spaces of constant curvature, Encyclopaedia of Math. Sci. 29, Springer-Verlag, 1993, 1-138. Zbl 0787.53001 MR 1254932

[A70] E. Andreev, On the intersection of the planes of faces of acute-angled polyhedra. Mat. Zametki 8 (1970), 521-527; English transl. Math. Notes 8 (1971), 761-764. Zbl 0209.26506 MR 0279680

[Bor] R. Borcherds, Automorphism groups of Lorentzian lattices. J. Algebra 111 (1987), 133-153. Zbl 0628.20003 MR 0913200 
Vol. $80(2005)$

[Cox] H. S. M. Coxeter, Regular honeycombs in hyperbolic space. In International Congress of Mathematicians, 1954, Amsterdam, vol. III, North-Holland Publishing Co., Amsterdam 1956, 155-169. Zbl 0073.36603 MR 0087114

[D] M. Davis, A hyperbolic 4-manifold. Proc. Amer. Math. Soc. 93 (1985), 325-328. Zbl 0533.51015 MR 0770546

[JS] T. Januszkiewicz and J. Swiatkowski, Hyperbolic Coxeter groups of large dimension. Comment. Math. Helv. 78 (2003), 555-583. Zbl 02021085 MR 1998394

[Kh] A. Khovanski, Hyperplane sections of polyhedra, toroidal manifolds, and discrete groups in Lobachevskii space. Funktsional. Anal. i Prilozhen. 20 (1986), 50-61; English transl. Functional Anal. Appl. 20 (1986), 41-50. Zbl 0597.51014

[N] V. Nikulin, On the classification of arithmetic groups generated by reflections in Lobachevsky spaces. Izv. Akad. Nauk SSSR Ser.Mat. 45 (1981), 113-142; English transl. Math. USSR-Izv. 18 (1982), 99-123. Zbl 0477.22008 MR 0607579

[Pr] M. Prokhorov, Absence of discrete reflexion groups with a non-compact polyhedron of finite volume in Lobachevsky spaces of large dimension. Izv. Akad. Nauk SSSR Ser. Mat. 50 (1986), 413-424; English transl. Math. USSR Izv. 28 (1987), 401-411. Zbl 0613.51010 MR 0842588

[Sc] P. Scott, Subgroups of surface groups are almost geometric. J. London Math. Soc. 17 (1978), 555-565; Correction ibid 32 (1985), 217-220. Zbl 0412.57006 Zbl 0581.57005 MR 0494062 MR 0811778

[Vi] E. B. Vinberg, Absence of crystallographic reflection groups in Lobachevsky space of large dimension. Trudy Moskov. Mat. Obshch. 47 (1984), 68-102; English transl. Trans. Mosc. Math. Soc. 1985, 75-112. Zbl 0593.22007 MR 0774946

[VS88] E. B. Vinberg and O. V. Shvartsman, Discrete groups of motions of spaces of constant curvature. Itogi Nauki Tekh., Ser. Sovrem. Probl. Mat., Fundam. Napravleniya 29 (1988), 147-259; English transl. in Geometry II. Spaces of Constant Curvature, Encyclopaedia of Math. Sci. 29, Springer-Verlag, Berlin 1993, 139-248. Zbl 0787.22012 MR 1254933

Received April 18, 2003

L. Potyagailo, UFR de Mathématiques, Université de Lille 1, 59655 Villeneuve d'Ascq cedex, France

E-mail: potyag@agat.univ-lille1.fr

E. Vinberg, Faculty of Mechanics and Mathematics, Moscow State University, Vorob'evy

Gory, Moscow 119992, GSP-2, Russia

E-mail: vinberg@zebra.ru 